\title{
Bio-potent (5-chloro-2-thienyl)-3-(substituted phenyl) bicyclo[2.2.1]heptane-2-yl methanone derivatives
}

\author{
G. Thirunarayanan \\ Department of Chemistry, Annamalai University, Annamalainagar 608 002, India \\ E-mail address: drgtnarayanan@gmail.com
}

\begin{abstract}
A series of (5-chloro-2-thienyl)(3-(substituted phenyl) bicyclo[2.2.1]hept-5-en-2-yl)methanones have been synthesized by fly-ash catalyzed [4+2] cycloaddition Diels-Alder reaction of cyclopentadiene and 5-chloro-2-thienyl chalcones under cooling conditions. The yields of the methanones are more than $60 \%$. The synthesized (5-chloro-2-thienyl)(3-(substituted phenyl) bicyclo[2.2.1]hept-5-en-2-yl)methanones are characterized by their physical constants and spectral data. The antimicrobial, antioxidant and insect antifeedant activities of synthesized methanones have been studied using their respective bacterial, fungal strains, DPPH radical scavenging activity and Dethler's leaf-discs bioassay method.
\end{abstract}

Keywords: Diels-Alder reaction; 5-chloro-2-thienyl chalcones; Cyclopentadiene; Antimicrobial activitiesl Antioxidant activity; Insect antifeedant activity

\section{INTRODUCTION}

Bicyclo [2.2.1] methanone compounds were synthesized by stereo selective [4+2] cycloaddition Diels-Alder reaction of diene and dienophiles [1]. In thermal condition this reaction is reversible and is known as retro-Diels-Alder reaction.

The mechanistic aspects of reactivity, selectivity, endo-exo- and solvent effects of this Diels-Alder reaction has been reported [2-6]. Currently, solvent-free Diels-Alder reaction is plays an important role for the synthesis of organic substrates especially bicyclo compounds with stereo selectivity, specificity, due to easy handling work-up and technical procedure, non-hazardousness, shorter reaction time, non-polluted to the environment and good yields $[1,7,8]$.

Reidant and Breslow[9] have studied the aqueous phase reaction of cyclopentadiene and vinyl methyl ketones in water and they reported the solvent-free reaction rate is greater than 700 times faster than in organic solvents. Numerous catalysts including Lewis acids [4], Bronsted acids [4,10], asymmetric catalyst with helical polymers [11], $\mathrm{Cu}^{2+}$ ion-mediated nanotubes [12], DNA and Micellar based catalysts [7,13-16] have been used for this [4+2] cycloaddition Diels-Alder reaction of cyclopentadiene(diene) and $E$ - chalcones(dienophiles).

The vinyl ketones, aza- vinyl ketones, bicyclo methanones possess important biological activities and antibodies [17].

The mono- or di- or tri- or poly $-\mathrm{OH}$ and $-\mathrm{OCH}_{3}$ substituted organic compounds possess significant antioxidant activities [18-20]. Similarly, mono- or di- or tri- or poly halogenated enones possess insect antifeedant activities [19-22]. Within the above view, there is no report 
available for the synthesis of (5-chloro-2-thienyl)(3-(substituted phenyl) bicyclo[2.2.1]hept-5en-2-yl)methanones by aqueous phase fly-ash catalyzed Diels-Alder reaction of cyclopentadiene and 4-chloro-1-aaphthyl chalcones under cooling condition.

Hence, the author have taken efforts to synthesize some (5-chloro-2-thienyl)(3(substituted phenyl) bicyclo[2.2.1]hept-5-en-2-yl)methanones and evaluated their pharmacological activities such as antimicrobial, antioxidant and insect antifeedant activities using their respective bacterial and fungal strains, DPPH radical scavenging [23] activeness and Dethler's [24] disc-diffusion bio-assay method.

\section{EXPERIMENTAL}

\section{1. General}

In this present investigation, all chemicals utilized were procured from Sigma-Aldrich and E-Merck brands. Fly ash was collected from Thermal Power Plant-II, Neyveli Lignite Corporation (NLC), Neyveli, Tamil Nadu, India.

Melting points of all bicyclo[2.2.1] heptene-2-yl methanones were determined in open glass capillaries on Mettler FP51 melting point apparatus and are uncorrected. Infrared spectra (KBr, 4000-400 $\mathrm{cm}^{-1}$ ) were recorded on Thermo scientific Nicolet iS5, US made Fourier transform spectrophotometer.

The NMR spectra of selective compounds were recorded in Bruker AV 400 spectrometer operating at $400 \mathrm{MHz}$ for ${ }^{1} \mathrm{H}$ NMR spectra and $100 \mathrm{MHz}$ for ${ }^{13} \mathrm{C}$ NMR spectra in $\mathrm{CDCl}_{3}$ solvent using TMS as internal standard. Electron impact and chemical ionization mode $\mathrm{FAB}^{+}$mass spectra were recorded with a SHIMADZU spectrometer.

\section{2. Synthesis of 5-chloro-2-thienyl chalcones}

The substituted styryl 5-chloro-2-thienyl ketones were synthesized by literature method [25].

\section{3. General procedure for synthesis of (5-chloro-2-thienyl)(3-(substituted phenyl) bicyclo[2.2.1]hept-5-en-2-yl)methanones}

Appropriate equi-molar quantities of 5-chloro-2-thienyl chalcones $(2 \mathrm{mmol})$ in $15 \mathrm{~mL}$ of ethanol, cyclopentadiene ( $2 \mathrm{mmol}$ ), and $0.4 \mathrm{~g}$ of fly-ash in $20 \mathrm{~mL}$ of water were stirred for $6 \mathrm{~h}$ in $0-4{ }^{\circ} \mathrm{C}$ (Scheme 1) and kept the reaction mixture for an overnight.

The completion of the reaction was monitored by thin layer chromatogram. Dichloromethane $(10 \mathrm{~mL})$ was added and extract was separated by filtration. The filtrate was washed with water, brine $(10 \mathrm{~mL})$, dried over on anhydrous $\mathrm{Na}_{2} \mathrm{SO}_{4}$ and concentrated gave the solid product.

The crude product was further purified by recrystallization with ethanol. 


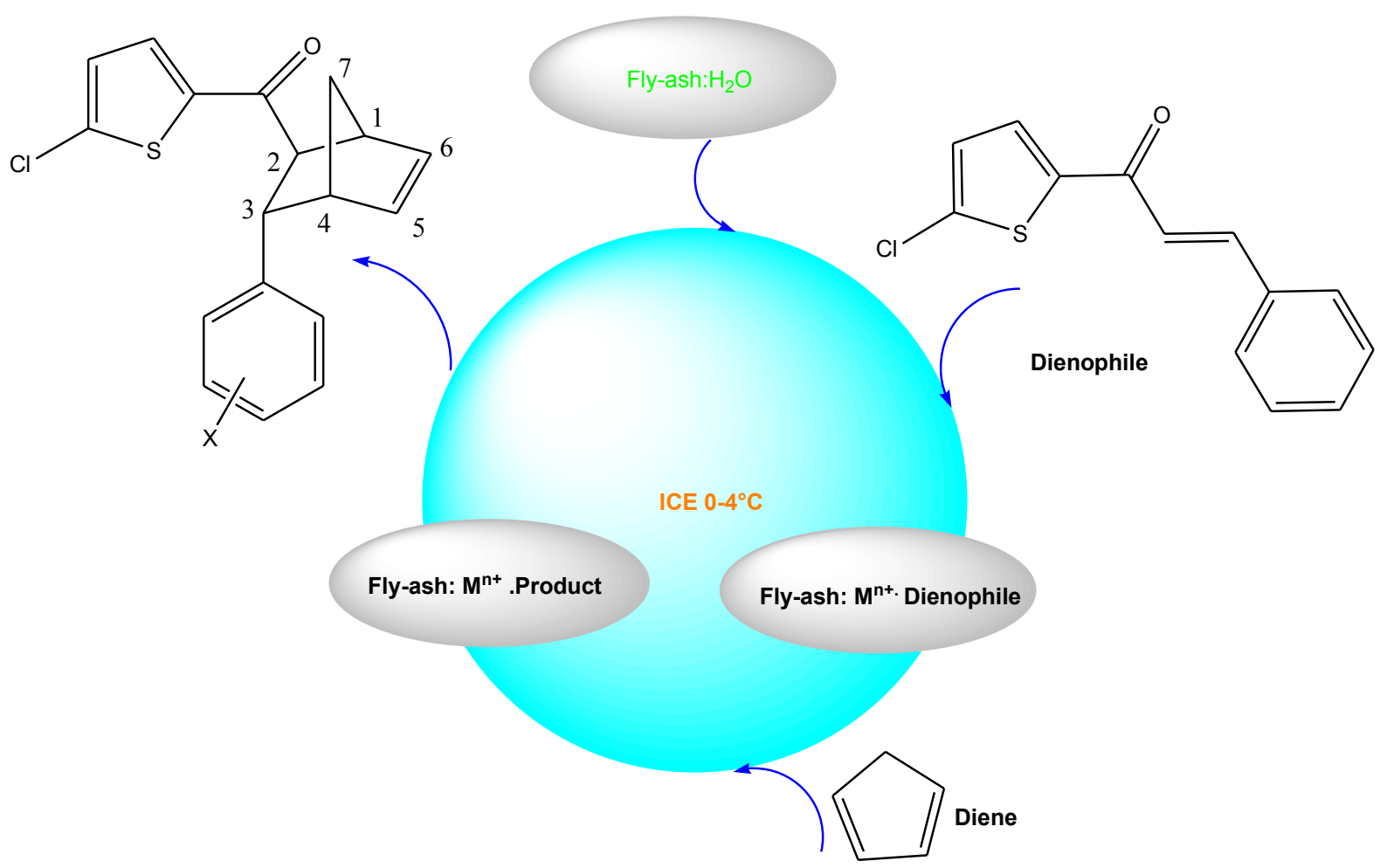

$\mathrm{X}=\mathrm{H}, 3-\mathrm{Br}, 3-\mathrm{Cl}, 2-\mathrm{F}, 4-\mathrm{F}, 4-\mathrm{OH}, 2-\mathrm{OCH}_{3}, 4-\mathrm{OCH}_{3}, 4-\mathrm{CH}_{3}, 4-\mathrm{NO}_{2}, 3-\mathrm{OC}_{6} \mathrm{H}_{5}$

Scheme 1. Synthesis of (5-chloro-2-thienyl)-3-(substituted phenyl)-bicyclo[2.2.1]hept-5-en-2-yl methanones by aqueous phase fly-ash catalyzed Diels-Alder reaction of 5-chloro-2-thienyl chalcones and cyclopentadiene,

\section{RESULTS AND DISCUSSION}

The author have attempts for the synthesis of aryl (5-chloro-2-thienyl)(3-(substituted phenyl) bicyclo[2.2.1]hept-5-en-2-yl)methanone derivatives by aqueous phase fly-ash catalyzed [4+2] cycloaddition Diels-Alder reaction with cyclopentadiene as diene and $E$ chalcones as dienophiles. Hence, the author have synthesized (5-chloro-2-thienyl)(3(substituted phenyl) bicyclo[2.2.1]hept-5-en-2-yl)methanones by aqueous phase Diels-Alder reaction of $(E)$-5-chloro-2-thienyl chalcones and cyclopentadiene under cooling condition. During the reaction the chemical species present in the fly-ash was catalyzed the $[4+2]$ cycloaddition of cyclopentadiene and $(E)$-5-chloro-2-thienyl chalcones. In this reaction the obtained yield is more than $60 \%$. The reusability of catalyst in this cycloaddition reaction was studied with $2 \mathrm{mmol}$ of 5-chloro-2-thienyl chalcone and $2 \mathrm{mmol}$ of cyclopentadiene (entry 1 ). The first run gave $65 \%$ of the product. The $2^{\text {nd }}$ and $3^{\text {rd }}$ runs gave 60 and $53 \%$. The fourth and fifth runs gave $40 \%$.

The chalcones containing electron donating substituents $\left(\mathrm{OCH}_{3}\right)$ gave higher yield than electron withdrawing (halogens and nitro) substituents. The effect of catalyst on this reaction was studied by varying the catalyst quantity from 0.1 to $0.5 \mathrm{~g}$. As the catalyst quantity increased from 0.1 to $0.4 \mathrm{~g}$ the percentage of product increased from $60-65 \%$. Further increase the catalyst amount beyond $0.4 \mathrm{~g}$, there is no increase in the percentage of product. The optimum quantity of catalyst loading was found to be $0.4 \mathrm{~g}$. The effect of solvents on this reaction (entry 1) was studied with the same quantity of reactants with methanol, 
dichloromethane, dioxane and tetrahydrofuran. In this solvent effect study, the observed yields are $65,63,62,60$ and $62 \%$ respectively. The higher yield was obtained in ethanol with fly-ash in water medium. The physical constants, infrared, NMR and mass spectral data of compounds are summarized as follows.

(5-chloro-2-thienyl)(3-phenylbicyclo[2.2.1]hept-5-en-2-yl)methanone 1:

Colourless solid, m.p. 102-103; IR(KBr) 2998, 1675, 1524, 1638, 1287, 1065, 927, 857, 634 $\mathrm{cm}^{-1} ;{ }^{1} \mathrm{H}$ NMR $\left(400 \mathrm{MHz}, \mathrm{CDCl}_{3}\right): \delta 2.579\left(\mathrm{dd}, 1 \mathrm{H}, \mathrm{H}_{1} ; J=4\right.$ and $\left.4.8 \mathrm{~Hz}\right), 2.764\left(\mathrm{t}, 1 \mathrm{H}, \mathrm{H}_{2} ; J\right.$ $=16 \mathrm{~Hz}), 3.150\left(\mathrm{t}, 1 \mathrm{H}, \mathrm{H}_{3} ; J=20 \mathrm{~Hz}\right), 2.687\left(\mathrm{dd}, 1 \mathrm{H}, \mathrm{H}_{4} ; J=6\right.$ and $\left.4 \mathrm{~Hz}\right), 5.712\left(\mathrm{~d}, 1 \mathrm{H}, \mathrm{H}_{5,6}, J\right.$ $=15 \mathrm{~Hz}), 1.990\left(\mathrm{dd}, 1 \mathrm{H}, \mathrm{H}_{7} ; J=4.8\right.$ and $\left.4 \mathrm{~Hz}\right), 1.742\left(\mathrm{dd}, 1 \mathrm{H}, \mathrm{H}_{7} ; J=8\right.$ and $\left.4 \mathrm{~Hz}\right), 6.723-$ 7.342(m, 7H, Ar-H); ${ }^{13} \mathrm{C}$ NMR( $\left.100 \mathrm{MHz}, \mathrm{CDCl}_{3}\right): \delta 193.82(\mathrm{CO}), 45.92\left(\mathrm{C}_{1}\right), \quad 52.33\left(\mathrm{C}_{2}\right)$, 48.72( $\left.\mathrm{C}_{3}\right), \quad 51.74\left(\mathrm{C}_{4}\right), 136.74\left(\mathrm{C}_{5,6}\right), \quad 47.83\left(\mathrm{C}_{7}\right), 125.25-147.72(\mathrm{Ar}-\mathrm{C})$; M.F. $\mathrm{C}_{18} \mathrm{H}_{15} \mathrm{ClOS}$;

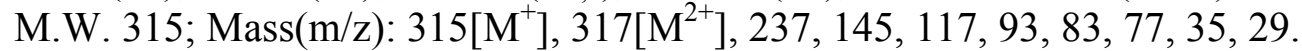

\section{3-(3-Bromophenyl)bicyclo[2.2.1]hept-5-en-2-yl)(5-chloro-2-thienyl)methanone 2:}

Pale yellow solid, m.p. 95-96; IR(KBr) 3005, 1649, 1574, 1468, 1329, 1084, 938, 726, 634 $\mathrm{cm}^{-1} ;{ }^{1} \mathrm{H}$ NMR $\left(400 \mathrm{MHz}, \mathrm{CDCl}_{3}\right): \delta 2.602\left(\mathrm{dd}, 1 \mathrm{H}, \mathrm{H}_{1} ; J=8\right.$ and $\left.4 \mathrm{~Hz}\right), 2.754\left(\mathrm{t}, 1 \mathrm{H}, \mathrm{H}_{2} ; J\right.$ $=16 \mathrm{~Hz}), 3.155\left(\mathrm{t}, 1 \mathrm{H}, \mathrm{H}_{3} ; J=16 \mathrm{~Hz}\right), 2.690\left(\mathrm{dd}, 1 \mathrm{H}, \mathrm{H}_{4} ; J=4\right.$ and $\left.4.8 \mathrm{~Hz}\right), 5.730\left(\mathrm{~d}, 1 \mathrm{H}, \mathrm{H}_{5,6}, J\right.$ $=15 \mathrm{~Hz}), 1.995\left(\mathrm{dd}, 1 \mathrm{H}, \mathrm{H}_{7} ; J=4\right.$ and $\left.6 \mathrm{~Hz}\right), 1.744\left(\mathrm{dd}, 1 \mathrm{H}, \mathrm{H}_{7} ; J=6\right.$ and $\left.4 \mathrm{~Hz}\right), 6.832-7.431(\mathrm{~m}$, $6 \mathrm{H}, \mathrm{Ar}-\mathrm{H}) ;{ }^{13} \mathrm{C} \mathrm{NMR}\left(100 \mathrm{MHz}, \mathrm{CDCl}_{3}\right): \delta$ 194.01(CO), 45.96( $\left.\mathrm{C}_{1}\right), 52.43\left(\mathrm{C}_{2}\right), 48.67\left(\mathrm{C}_{3}\right)$, 51.83( $\left.\mathrm{C}_{4}\right), 136.81\left(\mathrm{C}_{5,6}\right), 47.36\left(\mathrm{C}_{7}\right), 122.61-148.57(\mathrm{Ar}-\mathrm{C})$; M.F. $\mathrm{C}_{18} \mathrm{H}_{14} \mathrm{BrClOS}$; M.W. 394; $\operatorname{Mass}(\mathrm{m} / \mathrm{z}): 394\left[\mathrm{M}^{+}\right], 396\left[\mathrm{M}^{2+}\right], 398\left[\mathrm{M}^{4+}\right], 313,293,275,247,237,155,145,117,93,83,80$, $77,35,29$.

\section{3-(3-Chlorophenyl)bicyclo[2.2.1] hept-5-en-2-yl)(5-chloro-2-thienyl)methanone 3:}

Colour less yellow solid, m.p. 114-115; IR(KBr) 3010, 1628, 1528, 1493, 1086, 967, 827, 631 $\mathrm{cm}^{-1} ;{ }^{1} \mathrm{H}$ NMR $\left(400 \mathrm{MHz}, \mathrm{CDCl}_{3}\right): \delta 2.588\left(\mathrm{dd}, 1 \mathrm{H}, \mathrm{H}_{1} ; J=4\right.$ and $\left.6 \mathrm{~Hz}\right), 2.761\left(\mathrm{t}, 1 \mathrm{H}, \mathrm{H}_{2} ; J\right.$ $=16 \mathrm{~Hz}), 3.156\left(\mathrm{t}, 1 \mathrm{H}, \mathrm{H}_{3} ; J=16 \mathrm{~Hz}\right), 2.701\left(\mathrm{dd}, 1 \mathrm{H}, \mathrm{H}_{4} ; J=8\right.$ and $\left.4 \mathrm{~Hz}\right), 5.732\left(\mathrm{~d}, 2 \mathrm{H}, \mathrm{H}_{5,6}, J\right.$ $=15.5 \mathrm{~Hz}), 1.799\left(\mathrm{dd}, 1 \mathrm{H}, \mathrm{H}_{7} ; J=6\right.$ and $\left.4 \mathrm{~Hz}\right), 1.731\left(\mathrm{dd}, 1 \mathrm{H}, \mathrm{H}_{7} ; J=4.8\right.$ and $\left.4 \mathrm{~Hz}\right), 6.673-$ 7.403(m, $6 \mathrm{H}, \mathrm{Ar}-\mathrm{H}) ;{ }^{13} \mathrm{C} \mathrm{NMR}\left(100 \mathrm{MHz}, \mathrm{CDCl}_{3}\right): \delta 194.83(\mathrm{CO}), 45.93\left(\mathrm{C}_{1}\right), 52.38\left(\mathrm{C}_{2}\right)$, 48.52 $\left(\mathrm{C}_{3}\right)$, 51.32 $\left(\mathrm{C}_{4}\right), 136.74\left(\mathrm{C}_{5,6}\right), 47.27\left(\mathrm{C}_{7}\right), 125.55-147.76(\mathrm{Ar}-\mathrm{C}) ;$ M.F. $\mathrm{C}_{18} \mathrm{H}_{14} \mathrm{Cl}_{2} \mathrm{OS}$; M.W. 349; $\operatorname{Mass}(\mathrm{m} / \mathrm{z}): 349\left[\mathrm{M}^{+}\right], 351\left[\mathrm{M}^{2+}\right], 353\left[\mathrm{M}^{4+}\right], 313,237,189,161,145,117,111,93$, $77,35,29$.

\section{3-(2-Fluorophenyl)bicyclo[2.2.1]hept-5-en-2-yl)(5-chloro-2-thienyl)methanone 4:}

Colour less yellow solid, m.p. 103-104; IR(KBr) 3106, 1654, 1538, 1296, 1057, 934, 716, 628 $\mathrm{cm}^{-1} ;{ }^{1} \mathrm{H} \mathrm{NMR}\left(400 \mathrm{MHz}, \mathrm{CDCl}_{3}\right): \delta 2.602\left(\mathrm{dd}, 1 \mathrm{H}, \mathrm{H}_{1} ; J=6\right.$ and $\left.8 \mathrm{~Hz}\right), 2.743\left(\mathrm{t}, 1 \mathrm{H}, \mathrm{H}_{2} ; J=17\right.$ $\mathrm{Hz}), 3.157\left(\mathrm{t}, 1 \mathrm{H}, \mathrm{H}_{3} ; J=16 \mathrm{~Hz}\right), 2.698\left(\mathrm{dd}, 1 \mathrm{H}, \mathrm{H}_{4} ; J=4\right.$ and $\left.4.8 \mathrm{~Hz}\right), 6.153\left(\mathrm{~d}, 2 \mathrm{H}, \mathrm{H}_{5,6}, J=17\right.$ $\mathrm{Hz}), 2.001\left(\mathrm{dd}, 1 \mathrm{H}, \mathrm{H}_{7} ; J=8\right.$ and $\left.4 \mathrm{~Hz}\right), 1.732\left(\mathrm{dd}, 1 \mathrm{H}, \mathrm{H}_{7} ; J=4\right.$ and $\left.4.8 \mathrm{~Hz}\right), 6.638-7.432(\mathrm{~m}$, $6 \mathrm{H}, \mathrm{Ar}-\mathrm{H}) ;{ }^{13} \mathrm{C} \mathrm{NMR}\left(100 \mathrm{MHz}, \mathrm{CDCl}_{3}\right): \delta 194.58(\mathrm{CO}), 45.83\left(\mathrm{C}_{1}\right), 51.39\left(\mathrm{C}_{2}\right), 47.62\left(\mathrm{C}_{3}\right)$, 51.43( $\left(\mathrm{C}_{4}\right), 136.24\left(\mathrm{C}_{5,6}\right), 47.92\left(\mathrm{C}_{7}\right)$, 115.62-148.57(Ar-C); M.F. $\mathrm{C}_{18} \mathrm{H}_{14} \mathrm{ClFOS}$; M.W. 333; $\operatorname{Mass}(\mathrm{m} / \mathrm{z}): 333\left[\mathrm{M}^{+}\right], 335\left[\mathrm{M}^{2+}\right], 337\left[\mathrm{M}^{4+}\right], 313,297,187,145,117,111,93,77,35,29$.

\section{3-(4-Fluorophenyl)bicyclo[2.2.1]hept-5-en-2-yl)(5-chloro-2-thienyl)methanone 5:}

Pale yellow solid, m.p. 122-123; IR(KBr) 3062, 1659, 1526, 1234, 1039, $652 \mathrm{~cm}^{-1} ;{ }^{1} \mathrm{H}$ NMR $\left(400 \mathrm{MHz}, \mathrm{CDCl}_{3}\right): \delta 25.546\left(\mathrm{dd}, 1 \mathrm{H}, \mathrm{H}_{1} ; J=8\right.$ and $\left.6 \mathrm{~Hz}\right), 2.763\left(\mathrm{t}, 1 \mathrm{H}, \mathrm{H}_{2} ; J=19 \mathrm{~Hz}\right), 3.039(\mathrm{t}$, $\left.1 \mathrm{H}, \mathrm{H}_{3} ; J=16 \mathrm{~Hz}\right), 2.679\left(\mathrm{dd}, 1 \mathrm{H}, \mathrm{H}_{4} ; J=4\right.$ and $\left.4.8 \mathrm{~Hz}\right), 5.673\left(\mathrm{~d}, 1 \mathrm{H}, \mathrm{H}_{5,6} J=17 \mathrm{~Hz}\right), 2.004(\mathrm{dd}$, $1 \mathrm{H}, \mathrm{H}_{7} ; J=4$ and $\left.4.8 \mathrm{~Hz}\right), 1.731\left(\mathrm{dd}, 1 \mathrm{H}, \mathrm{H}_{7} ; J=4.8\right.$ and $\left.4 \mathrm{~Hz}\right), 6.732-7.321(\mathrm{~m}, 6 \mathrm{H}, \mathrm{Ar}-\mathrm{H}) ;{ }^{13} \mathrm{C}$ 
$\operatorname{NMR}\left(100 \mathrm{MHz}, \mathrm{CDCl}_{3}\right): \delta$ 195.08(CO), 45.83( $\left.\mathrm{C}_{1}\right), \quad 51.53\left(\mathrm{C}_{2}\right), \quad 47.66\left(\mathrm{C}_{3}\right), \quad 51.27\left(\mathrm{C}_{4}\right)$, 136.14( $\left(\mathrm{C}_{5,6}\right)$, 47.97( $\left.\mathrm{C}_{7}\right)$, 115.37-160.38(Ar-C); M.F. $\mathrm{C}_{18} \mathrm{H}_{14} \mathrm{ClFOS}$; M.W. 333; Mass(m/z): $333\left[\mathrm{M}^{+}\right], 335\left[\mathrm{M}^{2+}\right], 337\left[\mathrm{M}^{4+}\right], \quad 313,297,237,215,187,145,117,111,95,93,83,77,35,29$.

3-(4-Hydroxyphenyl)bicyclo[2.2.1]hept-5-en-2-yl)(5-chloro-2-thienyl)methanone 6:

Colour less solid, m.p. 98-100; IR(KBr) 3442, 3012, 1654, 1524, 1086, 934, 726, $627 \mathrm{~cm}^{-1}$; ${ }^{1} \mathrm{H}$ NMR $\left(400 \mathrm{MHz}, \mathrm{CDCl}_{3}\right): \delta 2.524\left(\mathrm{dd}, 1 \mathrm{H}, \mathrm{H}_{1} ; J=6.4\right.$ and $\left.4.8 \mathrm{~Hz}\right), 2.753\left(\mathrm{t}, 1 \mathrm{H}, \mathrm{H}_{2} ; J\right.$ $=18 \mathrm{~Hz}), 3.124\left(\mathrm{t}, 1 \mathrm{H}, \mathrm{H}_{3} ; J=17 \mathrm{~Hz}\right), 2.591\left(\mathrm{dd}, 1 \mathrm{H}, \mathrm{H}_{4} ; J=4.8\right.$ and $\left.4 \mathrm{~Hz}\right), 5.609\left(\mathrm{~d}, 1 \mathrm{H}, \mathrm{H}_{5,6}, J\right.$ $=16 \mathrm{~Hz}), 2.004\left(\mathrm{dd}, 1 \mathrm{H}, \mathrm{H}_{7} ; J=8\right.$ and $\left.4 \mathrm{~Hz}\right), 1.754\left(\mathrm{dd}, 1 \mathrm{H}, \mathrm{H}_{7} ; J=4\right.$ and $\left.4.8 \mathrm{~Hz}\right), 5.273(\mathrm{~s}, 1 \mathrm{H}$, $\mathrm{OH}), 6.653-7.341(\mathrm{~m}, 6 \mathrm{H}, \mathrm{Ar}-\mathrm{H}) ;{ }^{13} \mathrm{C} \mathrm{NMR}\left(100 \mathrm{MHz}, \mathrm{CDCl}_{3}\right): \delta 195.11(\mathrm{CO}), 45.83\left(\mathrm{C}_{1}\right)$, 51.50 $\left(\mathrm{C}_{2}\right), \quad 47.58\left(\mathrm{C}_{3}\right), \quad 51.27\left(\mathrm{C}_{4}\right), \quad 136.33\left(\mathrm{C}_{5,6}\right), \quad 47.98\left(\mathrm{C}_{7}\right), \quad 115.63-155.72(\mathrm{Ar}-\mathrm{C}) ; \quad$ M.F.

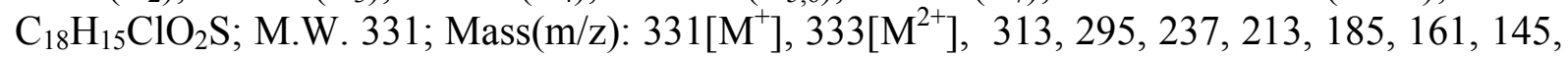
$127,117,93,83,77,35,29,17$.

3-(2-Methoxyphenyl)bicyclo[2.2.1]hept-5-en-2-yl)(5-chloro-2-thienyl)methanone 7:

Pale yellow solid, m.p. 122-123; IR(KBr) 3012, 2995, 1628, 1524, 1067, 928, $627 \mathrm{~cm}^{-1}$; ${ }^{1} \mathrm{H}$ NMR (400MHz, $\left.\mathrm{CDCl}_{3}\right): \delta 2.506\left(\mathrm{dd}, 1 \mathrm{H}, \mathrm{H}_{1} ; J=4\right.$ and $\left.4.8 \mathrm{~Hz}\right), 2.743\left(\mathrm{t}, 1 \mathrm{H}, \mathrm{H}_{2} ; J=18 \mathrm{~Hz}\right)$, $3.061\left(\mathrm{t}, 1 \mathrm{H}, \mathrm{H}_{3} ; J=17 \mathrm{~Hz}\right), 2.681\left(\mathrm{dd}, 1 \mathrm{H}, \mathrm{H}_{4} ; J=4\right.$ and $\left.6 \mathrm{~Hz}\right), 5.613\left(\mathrm{~d}, 1 \mathrm{H}, \mathrm{H}_{5,6}, J=18 \mathrm{~Hz}\right)$, $1.950\left(\mathrm{dd}, 1 \mathrm{H}, \mathrm{H}_{7} ; J=4\right.$ and $\left.4.8 \mathrm{~Hz}\right), 1.721\left(\mathrm{dd}, 1 \mathrm{H}, \mathrm{H}_{7} ; J=4.8\right.$ and $\left.4 \mathrm{~Hz}\right), 3.874(\mathrm{~s}, 3 \mathrm{H}$, $\left.\mathrm{OCH}_{3}\right), 6.973-7.331(\mathrm{~m}, 6 \mathrm{H}, \mathrm{Ar}-\mathrm{H}) ;{ }^{13} \mathrm{C} \mathrm{NMR}\left(100 \mathrm{MHz}, \mathrm{CDCl}_{3}\right): \delta 193.84(\mathrm{CO}), 45.79\left(\mathrm{C}_{1}\right)$, 51.46 $\left(\mathrm{C}_{2}\right), 47.63\left(\mathrm{C}_{3}\right)$, 51.32 $\left(\mathrm{C}_{4}\right), 136.07\left(\mathrm{C}_{5,6}\right), 47.83\left(\mathrm{C}_{7}\right)$, 59.76( $\left(\mathrm{OCH}_{3}\right), 113.74-161.73(\mathrm{Ar}-\mathrm{C})$; M.F. $\mathrm{C}_{19} \mathrm{H}_{17} \mathrm{ClO}_{2} \mathrm{~S}$; M.W. 345; $\operatorname{Mass}(\mathrm{m} / \mathrm{z}): 345\left[\mathrm{M}^{+}\right], 347\left[\mathrm{M}^{2+}\right], 329,237,145,117,93,91$, $83,77,35,31,29,15$.

\section{3-(4-Methoxyphenyl)bicyclo[2.2.1]hept-5-en-2-yl)(5-chloro-2-thienyl)methanone 8:}

Pale yellow solid, m.p. 116-117; IR(KBr) 2996, 1668, 1594, 1364, 1254, 1095, 924, 724, $634 \mathrm{~cm}^{-1} ;{ }^{1} \mathrm{H}$ NMR $\left(400 \mathrm{MHz}, \mathrm{CDCl}_{3}\right): \delta 2.510\left(\mathrm{dd}, 1 \mathrm{H}, \mathrm{H}_{1} ; J=4.8\right.$ and $\left.4 \mathrm{~Hz}\right), 2.753\left(\mathrm{t}, 1 \mathrm{H}, \mathrm{H}_{2}\right.$; $J=17 \mathrm{~Hz}), 3.173\left(\mathrm{t}, 1 \mathrm{H}, \mathrm{H}_{3} ; J=17 \mathrm{~Hz}\right), 2.632\left(\mathrm{dd}, 1 \mathrm{H}, \mathrm{H}_{4} ; J=8\right.$ and $\left.4 \mathrm{~Hz}\right), 5.608\left(\mathrm{~d}, 1 \mathrm{H}, \mathrm{H}_{5,6}, J\right.$ $=18 \mathrm{~Hz}), 1.953\left(\mathrm{dd}, 1 \mathrm{H}, \mathrm{H}_{7} ; J=4.8\right.$ and $\left.4 \mathrm{~Hz}\right), 1.743\left(\mathrm{dd}, 1 \mathrm{H}, \mathrm{H}_{7} ; J=8\right.$ and $\left.4 \mathrm{~Hz}\right), 3.867(\mathrm{~s}, 3 \mathrm{H}$, $\left.\mathrm{OCH}_{3}\right), 6.745-7.438(\mathrm{~m}, 6 \mathrm{H}, \mathrm{Ar}-\mathrm{H}) ;{ }^{13} \mathrm{C} \mathrm{NMR}\left(100 \mathrm{MHz}, \mathrm{CDCl}_{3}\right): \delta 193.53(\mathrm{CO}), 45.77\left(\mathrm{C}_{1}\right)$, 51.54( $\left(\mathrm{C}_{2}\right), 47.83\left(\mathrm{C}_{3}\right), 51.43\left(\mathrm{C}_{4}\right), 136.09\left(\mathrm{C}_{5,6}\right), 47.99\left(\mathrm{C}_{7}\right), 58.96\left(\mathrm{OCH}_{3}\right), 113.98-158.83(\mathrm{Ar}-\mathrm{C})$; M.F. $\mathrm{C}_{19} \mathrm{H}_{17} \mathrm{ClO}_{2} \mathrm{~S}$; M.W. 345; $\operatorname{Mass}(\mathrm{m} / \mathrm{z}): 345\left[\mathrm{M}^{+}\right], 347\left[\mathrm{M}^{2+}\right], 329,313,294,237,227,145$, $117,107,93,91,83,77,35,31,29,15$.

\section{3-(4-Methylphenyl)bicyclo[2.2.1]hept-5-en-2-yl)(5-chloro-2-thienyl)methanone 9:}

Colour less solid, m.p. 105-106; IR(KBr) 2998, 1638, 1524, 1329, 1264, 1061, 928, 824, 631 $\mathrm{cm}^{-1} ;{ }^{1} \mathrm{H}$ NMR $\left(400 \mathrm{MHz}, \mathrm{CDCl}_{3}\right): \delta 2.589\left(\mathrm{dd}, 1 \mathrm{H}, \mathrm{H}_{1} ; J=4\right.$ and $\left.4.8 \mathrm{~Hz}\right), 2.716\left(\mathrm{t}, 1 \mathrm{H}, \mathrm{H}_{2}\right.$; $J=19 \mathrm{~Hz}), 3.091\left(\mathrm{t}, 1 \mathrm{H}, \mathrm{H}_{3} ; J=18 \mathrm{~Hz}\right), 2.679\left(\mathrm{dd}, 1 \mathrm{H}, \mathrm{H}_{4} ; J=4\right.$ and $\left.4.8 \mathrm{~Hz}\right), 5.617\left(\mathrm{~d}, 1 \mathrm{H}, \mathrm{H}_{5,6}, J\right.$ $=17 \mathrm{~Hz}), 1.954\left(\mathrm{dd}, 1 \mathrm{H}, \mathrm{H}_{7} ; J=4\right.$ and $\left.4.8 \mathrm{~Hz}\right), 1.745\left(\mathrm{dd}, 1 \mathrm{H}, \mathrm{H}_{7} ; J=6\right.$ and $\left.4 \mathrm{~Hz}\right), 2.417(\mathrm{~s}, 3 \mathrm{H}$, $\left.\mathrm{CH}_{3}\right), 6.782-7.431(\mathrm{~m}, 6 \mathrm{H}, \mathrm{Ar}-\mathrm{H}) ;{ }^{13} \mathrm{C} \mathrm{NMR}\left(100 \mathrm{MHz}, \mathrm{CDCl}_{3}\right): \delta 196.21(\mathrm{CO}), 45.78\left(\mathrm{C}_{1}\right)$, 51.62 $\left(\mathrm{C}_{2}\right), 47.88\left(\mathrm{C}_{3}\right)$, 51.22( $\left.\mathrm{C}_{4}\right), 135.92\left(\mathrm{C}_{5,6}\right), 48.23\left(\mathrm{C}_{7}\right), 27.32\left(\mathrm{CH}_{3}\right), 126.98-143.57(\mathrm{Ar}-\mathrm{C})$; M.F. $\mathrm{C}_{19} \mathrm{H}_{17} \mathrm{ClOS}$; M.W. 329; Mass(m/z): 329[M $\left[\mathrm{M}^{+}\right], 331\left[\mathrm{M}^{2+}\right], 313,293,237,211,183,161$, $145,127,117,93,91,83,77,35,29,15$.

\section{3-(4-Nitrophenyl)bicyclo[2.2.1]hept-5-en-2-yl)(5-chloro-2-thienyl)methanone 10:}

Pale yellow solid, m.p. 111-112; IR(KBr) 3016, 1679, 1597, 1087, 864, 794, $691 \mathrm{~cm}^{-1} ;{ }^{1} \mathrm{H}$ NMR (400MHz, $\left.\mathrm{CDCl}_{3}\right): \delta 2.6074\left(\mathrm{dd}, 1 \mathrm{H}, \mathrm{H}_{1} ; J=8\right.$ and $\left.4 \mathrm{~Hz}\right), 2.863\left(\mathrm{t}, 1 \mathrm{H}, \mathrm{H}_{2} ; J=15 \mathrm{~Hz}\right)$, $3.165\left(\mathrm{t}, 1 \mathrm{H}, \mathrm{H}_{3} ; J=16 \mathrm{~Hz}\right), 2.730\left(\mathrm{dd}, 1 \mathrm{H}, \mathrm{H}_{4} ; J=4\right.$ and $\left.4.8 \mathrm{~Hz}\right), 5.699\left(\mathrm{~d}, 1 \mathrm{H}, \mathrm{H}_{5,6}, J=17 \mathrm{~Hz}\right)$, 
$1.928\left(\mathrm{dd}, 1 \mathrm{H}, \mathrm{H}_{7} ; J=8\right.$ and $\left.4 \mathrm{~Hz}\right), 1830\left(\mathrm{dd}, 1 \mathrm{H}, \mathrm{H}_{7} ; J=4.8\right.$ and $\left.4 \mathrm{~Hz}\right), 6.831-8.124(\mathrm{~m}, 6 \mathrm{H}$, $\mathrm{Ar}-\mathrm{H}) ;{ }^{13} \mathrm{C} \mathrm{NMR}\left(100 \mathrm{MHz}, \mathrm{CDCl}_{3}\right): \delta 196.98(\mathrm{CO}), 45.93\left(\mathrm{C}_{1}\right), 52.63\left(\mathrm{C}_{2}\right), 47.63\left(\mathrm{C}_{3}\right)$, 51.72 $\left(\mathrm{C}_{4}\right)$, 136.97( $\left(\mathrm{C}_{5,6}\right), 48.24\left(\mathrm{C}_{7}\right), 121.78-152.83(\mathrm{Ar}-\mathrm{C})$; M.F. $\mathrm{C}_{18} \mathrm{H}_{14} \mathrm{ClNO}_{3} \mathrm{~S} ;$ M.W. 360; $\operatorname{Mass}(\mathrm{m} / \mathrm{z}): 360\left[\mathrm{M}^{+}\right], 362\left[\mathrm{M}^{2+}\right], 324,313,279,242,237,203,197,145,122,117,110,93,83$, $77,46,35,29$.

3-(3-Phenoxyphenyl)bicyclo[2.2.1] hept-5-en-2-yl)(5-chloro-2-thienyl)methanone 11:

Colour less solid, m.p. 122-123; IR(KBr) 3016, 1654, 1539, 1058, 827, 764, 639 $\mathrm{cm}^{-1} ;{ }^{1} \mathrm{H}$ $\mathrm{NMR}\left(400 \mathrm{MHz}, \mathrm{CDCl}_{3}\right): \delta 2.250\left(\mathrm{dd}, 1 \mathrm{H}, \mathrm{H}_{1} ; J=4.8\right.$ and $\left.4 \mathrm{~Hz}\right), 2.839\left(\mathrm{t}, 1 \mathrm{H}, \mathrm{H}_{2} ; J=17 \mathrm{~Hz}\right)$, 3.091(t, $\left.1 \mathrm{H}, \mathrm{H}_{3} ; J=16 \mathrm{~Hz}\right), 2.714\left(\mathrm{dd}, 1 \mathrm{H}, \mathrm{H}_{4} ; J=4\right.$ and $\left.4.8 \mathrm{~Hz}\right), 5.683\left(\mathrm{~d}, 1 \mathrm{H}, \mathrm{H}_{5,6}, J=17 \mathrm{~Hz}\right)$, $1.999\left(\mathrm{dd}, 1 \mathrm{H}, \mathrm{H}_{7} ; J=8\right.$ and $\left.4 \mathrm{~Hz}\right), 1.788\left(\mathrm{dd}, 1 \mathrm{H}, \mathrm{H}_{7} ; J=4\right.$ and $\left.4.8 \mathrm{~Hz}\right), 6.156-7.453(\mathrm{~m}, 11 \mathrm{H}$, $\mathrm{Ar}-\mathrm{H}) ;{ }^{13} \mathrm{C} \mathrm{NMR}\left(100 \mathrm{MHz}, \mathrm{CDCl}_{3}\right): \delta 194.57(\mathrm{CO}), 45.82\left(\mathrm{C}_{1}\right), 52.46\left(\mathrm{C}_{2}\right), 47.27\left(\mathrm{C}_{3}\right)$, 51.19( $\left.\mathrm{C}_{4}\right), 136.22\left(\mathrm{C}_{5,6}\right), 46.36\left(\mathrm{C}_{7}\right), 114.23-146.37(\mathrm{Ar}-\mathrm{C})$; M.F. $\mathrm{C}_{24} \mathrm{H}_{19} \mathrm{ClOS} ;$ M.W. 390; $\operatorname{Mass}(\mathrm{m} / \mathrm{z}): 390\left[\mathrm{M}^{+}\right], 392\left[\mathrm{M}^{2+}\right], 355,313,279,245,237,153,145,117,110,93,83,77,46$, $35,29$.

\section{1. Antimicrobial activities}

The antimicrobial activities of synthesized (5-chloro-2-thienyl)(3-(substituted phenyl) bicyclo[2.2.1]hept-5-en-2-yl)methanones have been evaluated by measuring the mm of zone of inhibition of the compounds against the bacterial and fungal strains. In this present investigations, the author have chosen two gram positive pathogenic strains Staphylococcus aureus, Entrocccus faecalis while Escherichia coli, Klebsiella species, Psuedomonas and Proteus vulgaris were the gram negative strains. The disc diffusion technique was followed using the Kirby-Bauer [26] method, at a concentration of $250 \mu \mathrm{g} / \mathrm{mL}$ with Ampicillin and Streptomycin taken as the standard drugs. For the study of antifungal activities of all methanones using Candida albicans as the fungal strain and the disc diffusion technique was followed for the antifungal activity while the two other stains Penicillium species and Aspergillus niger, the dilution method will be used. The drugs dilution will be $50 \mu \mathrm{g} / \mathrm{mL}$. Grisseofulvin is taken as the standard drug.

\section{1. 1. Antibacterial sensitivity assay}

Antibacterial sensitivity assay of (5-chloro-2-thienyl)(3-substituted phenyl bicyclo [2.2.1]hept-5-en-2-yl)methanones were performed using Kirby-Bauer [26] disc diffusion technique. In each petri plate about $0.5 \mathrm{~mL}$ of the test bacterial sample was spread uniformly over the solidified Mueller Hinton agar using sterile glass spreader. Then the discs with $5 \mathrm{~mm}$ diameter made up of Whatmann No.1 filter paper, impregnated with the solution of the compound were placed on the medium using sterile forceps. The plates were incubated for 24 $\mathrm{h}$ at $37^{\circ} \mathrm{C}$ by keeping the plates upside down to prevent the collection of water droplets over the medium. After $24 \mathrm{~h}$, the plates were visually examined and the diameter values of the zone of inhibition were measured. Triplicate results were recorded by repeating the same procedure.

The observed antibacterial activities of all (5-chloro-2-thienyl)(3-substituted phenyl bicyclo[2.2.1]hept-5-en-2-yl)methanones were presented in Table 1. Almost all compounds shows antibacterial activity. Methanones 2-5 and 9 were shown maximum zone of inhibition against Escherichia coli, with greater than $20 \mathrm{~mm}$ of zone of inhibition compared to the ketones $\mathbf{1}$ and $\mathbf{1 1}$ are active in 13-19 mm of zone of inhibition. 
Table 1. Antibacterial ${ }^{\mathrm{a}}$, antifungal ${ }^{\mathrm{b}}$ and antioxidant ${ }^{\mathrm{c}}$ activities of (5-chloro-2-thienyl)-3-(substituted phenyl)-bicyclo[2.2.1]hept-5-en-2-yl methanones.

\begin{tabular}{|c|c|c|c|c|c|c|c|c|c|c|c|}
\hline \multirow[b]{2}{*}{ Entry } & \multirow[b]{2}{*}{$\mathrm{X}$} & \multicolumn{6}{|c|}{ Antibacterial activity } & \multicolumn{3}{|c|}{ Antifungal activity } & \multirow[b]{2}{*}{$\begin{array}{l}\text { Antioxidant } \\
\text { activity } \\
\text { (DPPH } \\
\text { radical } \\
\text { scavenging) }\end{array}$} \\
\hline & & 隹 & is & 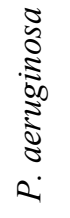 & 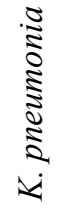 & 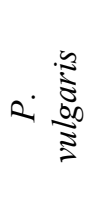 & 证莺 & ن ن & 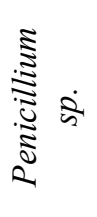 & 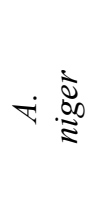 & \\
\hline 1 & $I$ & + & + & \pm & \pm & \pm & \pm & \pm & \pm & \pm & 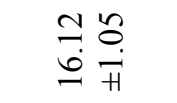 \\
\hline 2 & 离 & ++ & ++ & ++ & ++ & ++ & ++ & + & + & \pm & 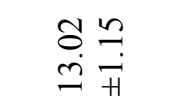 \\
\hline 3 & $\begin{array}{l}\underbrace{}_{1} \\
m\end{array}$ & ++ & ++ & ++ & ++ & ++ & ++ & \pm & \pm & + & $\begin{array}{c}\text { mb } \\
\infty \\
\infty\end{array}$ \\
\hline 4 & II & ++ & ++ & ++ & ++ & ++ & + & \pm & \pm & \pm & 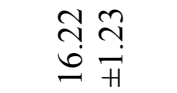 \\
\hline 5 & $\frac{1}{\dot{g}}$ & ++ & ++ & ++ & ++ & ++ & + & \pm & \pm & \pm & \begin{tabular}{l}
$\simeq 6$ \\
\hdashline \\
0 \\
0
\end{tabular} \\
\hline 6 & 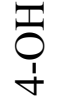 & \pm & + & + & \pm & \pm & + & + & \pm & + & 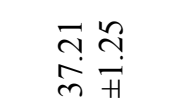 \\
\hline 7 & 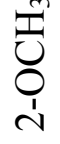 & \pm & + & + & + & ++ & ++ & ++ & + & ++ & 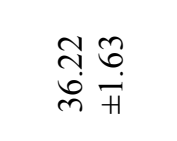 \\
\hline 8 & 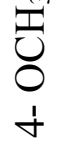 & \pm & ++ & ++ & ++ & + & ++ & ++ & ++ & ++ & 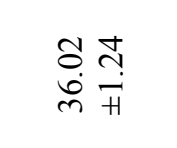 \\
\hline 9 & 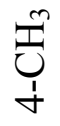 & ++ & \pm & ++ & ++ & + & \pm & + & + & + & 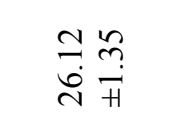 \\
\hline
\end{tabular}




\begin{tabular}{|c|c|c|c|c|c|c|c|c|c|c|c|}
\hline 10 & $\overbrace{\substack{Z \\
\dot{Z}}}^{N}$ & \pm & \pm & + & + & + & \pm & ++ & ++ & ++ & 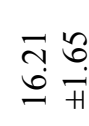 \\
\hline 11 & 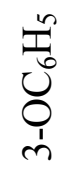 & + & + & \pm & \pm & ++ & + & + & ++ & ++ & $\stackrel{+}{m} \stackrel{n}{m}$ \\
\hline
\end{tabular}

${ }^{\text {a} D i s c ~ s i z e: ~} 6.35 \mathrm{~mm}$; Duration: 24-45 h; Standard: Ampicillin (30-33 mm) and Streptomycin(20-25 mm); Control: Methanol; ---: No activities; \pm : Active(8-12 mm); +: Moderately active(13-19 mm); ++: Active(20-24 $\mathrm{mm})$.

${ }^{\mathrm{b}}$ Standard: Griseofulvin and Gentamycin; Duration : 72 h; Control: Methanol; Medium: Potato dextrose agar; ++: No fungal colony; +: One fungal colony; \pm : Two-three fungal colonies; ---: Heavy fungal colony. 'Sandard: $\alpha$-Tocoferol (37.34 \pm 1.57$)$.

Compounds 6-8 and $\mathbf{1 0}$ are moderately active within 8-12 $\mathrm{mm}$ of zone of inhibition. The methanone derivatives $\mathbf{2 - 5 , 7}$ and $\mathbf{8}$ were found to be effective against $S$. aureus within 20-24 $\mathrm{mm}$ of zone of inhibition. Compounds $\mathbf{1 ,} \mathbf{6}$ and $\mathbf{1 1}$ were active within 13-19 mm of zone of inhibition. The methanone derivatives 9 and $\mathbf{1 0}$ were moderately active within $8-12 \mathrm{~mm}$ of zone of inhibition. The methanone derivatives $2-5,8$ and 9 were more active against Pseudomonas showing greater than $20 \mathrm{~mm}$ zone of inhibition and the other derivatives $\mathbf{6 , 7}$ and 10 were showed the zone of inhibitions between 13-19 mm. Compounds 1 and 11 has shown moderately active with the zone of inhibition of 8-12 $\mathrm{mm}$.

The ketones 2-5, 8 and 9 are effective against Klebsiella with 20-24 mm zone of inhibition while the other ketones $\mathbf{7}$ and $\mathbf{1 0}$ showed an active and the compounds $\mathbf{1 ,} \mathbf{6}$ and $\mathbf{1 1}$ are possess moderate activity. The methanones 2-5, 7 and $\mathbf{1 1}$ were active when it is screened against $P$. vulgaris. The compounds 8, $\mathbf{9}$ and $\mathbf{1 0}$ are active against $3-19 \mathrm{~mm}$ of zone of inhibition and the other compounds 1 and $\mathbf{6}$ are moderately effective. The ketones 2, 3, 7 and 8 showed activities against E-faecalis when they are screened with 20-24 mm zone of inhibition. The ketones 4-6 and $\mathbf{1 1}$ are active against 13-19 $\mathrm{mm}$ of zone of inhibition. The methanones 1, 9 and $\mathbf{1 0}$ are moderately active.

\section{1. 2. Antifungal sensitivity assay}

Antifungal activity of synthesized (5-chloro-2-thienyl)(3-substituted phenyl bicyclo [2.2.1] hept-5-en-2-yl)methanone were performed using Kirby-Bauer[26] disc diffusion technique. PDA medium was prepared and sterilized as above. It was poured (ear bearing heating condition) in the Petri-plate which was already filled with $1 \mathrm{~mL}$ of the fungal species. The plate was rotated clockwise and counter clock-wise for uniform spreading of the species. The discs were impregnated with the test solution. The test solution was prepared by dissolving $15 \mathrm{mg}$ of the methanone in $1 \mathrm{~mL}$ of DMSO solvent. The medium was allowed to solidify and kept for $24 \mathrm{~h}$. Then the plates were visually examined and the diameter values of zone of inhibition were measured. Triplicate results were recorded by repeating the same procedure.

The observed antifungal activities of all prepared methanones are presented in Table 1. The study of antifungal activities of all methanones against $C$. albicans, showed that the three compounds 7, 8 and $\mathbf{1 0}$ are effective with $20 \mathrm{~mm}$ as the zone of inhibition in $250 \mu \mathrm{g} / \mathrm{mL}$ while 
methanones 2, 6, 9 and $\mathbf{1 1}$ are active with 13-19 mm zone of inhibition and the compound $\mathbf{1}$ and 3-5 were least active with 8-12 mm zone of inhibitions. Compounds 8, 10 and $\mathbf{1 1}$ are visible against Penicillum species, in the development of the absence of fungal colony and the compounds 2, 7 and 9 shows active within 13-19 $\mathrm{mm}$ of zone of inhibition. The methanones 1 and 3-6 are moderately active in 8-12 $\mathrm{mm}$ of zone of inhibition.

The inhibition of ketones against A.niger was less in one compound 7, 8, 10 and 11 being highly active followed by 3,6 and 9 . The ketone 1, 2, 4 and 7 were moderately active with production of one fungal colony. Presence of a bromo, chloro, methoxy, methyl and nitro substituents are responsible for antimicrobial activities of methanones.

\subsection{Antioxidant activity}

The antioxidant activities of synthesized (5-chloro-2-thienyl)(3-substituted phenyl bicyclo[2.2.1]hept-5-en-2-yl)methanones have been evaluated by the DPPH radical scavenging effect [27]. The $0.1 \mathrm{M}$ acetate was prepared by dissolving $1.64 \mathrm{~g}$ of sodium acetate in $15 \mathrm{~mL}$ of water and $150 \mu \mathrm{L}$ of acetic acid.

The final volume was adjusted to $20 \mathrm{~mL}$ by adding water. The $0.2 \mathrm{mmol}$ of DPPH solution was prepared by dissolving $3.9 \mathrm{~g}$ of DPPH in $50 \mathrm{~mL}$ of ethanol. $\alpha$-Tocofereol $(1 \mathrm{mg}$ in $10 \mathrm{~mL}$ of ethanol) solution was prepared. A series of test tubes were arranged with $1.0 \mathrm{~mL}$ of buffer solution mixed with $0.5 \mathrm{~mL}$ of DPPH solution. A series of various concentrations of synthesized methanones and $\alpha$-Tocofereol $(1 \mu \mathrm{g}$ in $1 \mathrm{ml}$ of ethanol) was added to each tube and mixed well. After 30 minutes in room temperature the absorbance of each solution was measured by UV spectrophotometer at $517 \mathrm{~nm}$.

A mixture of buffer solution and ethanol used as the reference for the spectrophotometer. A graph was plotted with the weight of the compound Vs absorptions and IC50 values will be determined. The antioxidant activity was expressed in terms of IC50 $(\mu \mathrm{g} / \mathrm{mL}$, concentration required to inhibit DPPH radical formation by $50 \%$ ). $\alpha$-Tocofereol will be used as a positive control. The radical scavenging activity was calculated as,

DPPH radical scavenging activity $=\frac{\text { Control absorbance }- \text { Sample absorbance }}{\text { Control absorbance }} \times 100$
$(\%$ of inhibition $)$

From the experimental statistical results, the observed antioxidant activities of methanones were presented in Table 1. From Table 1, the hydroxy and methoxy substituted methanones $(6,7$ and 8$)$ were shown significant antioxidant activity.

\section{3. Insect antifeedant activity}

Generally organic compounds which are having carbonyl, unsaturation and halogen substitutions, they possess insect antifeedant activity. Therefore, the author wishes to examine the insect antifeedant activity of these (5-chloro-2-thienyl)(3-substituted phenyl bicyclo[2.2.1]hept-5-en-2-yl)methanones and found to be active as insect antifeedants.

This test was performed with a $4^{\text {th }}$ instar larva Achoea Janata L against castor semilooper, were reared as described on the leaves of castor, Ricinus communis in the laboratory at the temperature range of $26{ }^{\circ} \mathrm{C} \pm 1{ }^{\circ} \mathrm{C}$ and a relative humidity of $75-85 \%$. The leaf - disc bioassay method [20,21] was used against the $4^{\text {th }}$ instar larvae to measure the antifeedant activity. The $4^{\text {th }}$ instar larvae were selected for testing because the larvae at this stage feed very voraciously. 


\section{3. 1. Measurement of insect antifeedant activity of (5-chloro-2-thienyl)(3-substituted phenyl bicyclo[2.2.1]hept-5-en-2-yl)methanones}

Castor leaf discs of a diameter of $1.85 \mathrm{~cm}$ were punched and intact with the petioles. Synthesized (5-chloro-2-thienyl)(3-substituted phenyl bicyclo[2.2.1]hept-5-en-2-yl) methanones were dissolved in acetone at a concentration of 200 ppm dipped for 5 minutes. The leaf discs were air-dried and placed in one liter beaker containing little water in order to facilitate translocation of water. Therefore, the leaf discs remain fresh throughout the duration of the rest, $4^{\text {th }}$ instar larvae of the test insect, which had been preserved on the leaf discs of all bicyclo [2.2.1] hepane-2-yl methanones and allowed to feed on them for $24 \mathrm{~h}$. The areas of the leaf disc consumed were measured by Dethler's method [24]. The observed antifeedant activity of (5-chloro-2-thienyl)(3-substituted phenyl bicyclo[2.2.1]hept-5-en-2-yl)methanones was presented in Table 2.

The results of the antifeedant activity of bicyclo[2.2.1] heptane-2-yl methanones are presented in Table 2 reveals that compound 3 (3-(3-chlorophenyl)bicyclo[2.2.1] hept-5-en-2yl)(5-chloro-2-thienyl)methanone was found to reflect significant antifeedant activity. This test is performed with the insects which ate only two-leaf disc soaked under the solution of this compound. Compounds 2, 4 and 5 showed enough antifeedant activity but lesser than 3. Further compound $\mathbf{3}$ was subjected to measure the antifeedant activity at different 50, 100, $150 \mathrm{ppm}$ concentrations and the observation reveals that as the concentrations decreased, the activity also decreased. It is observed from the results in Table 3 and that the compound $\mathbf{3}$ (3(3-chlorophenyl) bicyclo [2.2.1]hept-5-en-2-yl)(5-chloro-2-thienyl) methanone showed an appreciable antifeedant activity at $150 \mathrm{ppm}$ concentration.

Table 2. The insect antifeedant activities of (5-chloro-2-thienyl)-3-(substituted phenyl)bicyclo[2.2.1]hept-5-en-2-yl methanones.

\begin{tabular}{|c|c|c|c|c|c|c|c|c|c|c|c|}
\hline Entry & $\mathrm{R}$ & $\begin{array}{c}4-6 \\
\mathrm{pm}\end{array}$ & $\begin{array}{c}6-8 \\
\mathrm{pm}\end{array}$ & $\begin{array}{c}8-10 \\
\mathrm{pm}\end{array}$ & $\begin{array}{c}10 \mathrm{pm}- \\
12 \mathrm{am}\end{array}$ & $\begin{array}{c}12-6 \\
\mathrm{am}\end{array}$ & $\begin{array}{c}6-8 \\
\mathrm{am}\end{array}$ & $\begin{array}{c}8 \mathrm{am}- \\
12 \mathrm{Nn}\end{array}$ & $\begin{array}{c}12 \mathrm{Nn}- \\
2 \mathrm{pm}\end{array}$ & $\begin{array}{c}2-4 \\
\mathrm{pm}\end{array}$ & $\begin{array}{c}\text { Total leaf } \\
\text { disc } \\
\text { consumed } \\
\text { by } 24 \mathrm{~h}\end{array}$ \\
\hline $\mathbf{1}$ & $\mathrm{H}$ & 1 & 0.5 & 1 & 0.5 & 0.5 & 1 & 1 & 1 & 1 & 7 \\
\hline $\mathbf{2}$ & $3-\mathrm{Br}$ & 0.5 & 0.5 & 1 & 1 & 1 & 0 & 0 & 0 & 0 & 4 \\
\hline $\mathbf{3}$ & $3-\mathrm{Cl}$ & 0.5 & 0 & 0 & 0.5 & 0.5 & 0.5 & 0 & 1 & 0 & 3.5 \\
\hline $\mathbf{4}$ & $2-\mathrm{F}$ & 1 & 0 & 0.5 & 0 & 0 & 0 & 1 & 1 & 1 & 4.5 \\
\hline $\mathbf{5}$ & $4-\mathrm{F}$ & 1 & 0.5 & 0.5 & 0 & 0 & 0 & 0.5 & 0.5 & 0.5 & 4 \\
\hline $\mathbf{6}$ & $4-\mathrm{OH}$ & 1 & 1 & 2 & 0.25 & 0.25 & 0 & 0 & 1 & 0.5 & 6.5 \\
\hline $\mathbf{7}$ & $2-\mathrm{OCH}_{3}$ & 1 & 1 & 0 & 1 & 1 & 1 & 1 & 1 & 1 & 8 \\
\hline $\mathbf{8}$ & $4-\mathrm{OCH}_{3}$ & 1 & 0.5 & 1 & 1 & 1 & 0.5 & 1 & 0.5 & 0.5 & 7 \\
\hline $\mathbf{9}$ & $4-\mathrm{CH}_{3}$ & 2 & 1 & 0 & 1 & 1 & 1 & 0 & 1 & 1 & 8 \\
\hline $\mathbf{1 0}$ & $4-\mathrm{NO}_{2}$ & 1 & 2 & 1 & 1 & 0.5 & 0.5 & 0.5 & 0.5 & 1 & 9 \\
\hline $\mathbf{1 1}$ & $3-\mathrm{OC}_{6} \mathrm{H}_{5}$ & 1 & 1 & 1 & 1 & 1 & 1 & 0 & 1 & 1 & 8 \\
\hline
\end{tabular}

Number of leaf discs consumed by the insect (values are mean + SE of five). 
Table 3. Antifeedant activity of compound 3 (5-chloro-2-thienyl)-3-(3-chlorophenyl)bicyclo[2.2.1] hept-5-en-2-yl methanone showed an appreciable antifeedant activity at 3 different concentrations.

\begin{tabular}{|l|l|l|l|l|l|l|l|l|l|l|}
\hline $\mathrm{ppm}$ & $\begin{array}{l}4-6 \\
\mathrm{pm}\end{array}$ & $\begin{array}{l}6-8 \\
\mathrm{pm}\end{array}$ & $\begin{array}{l}8-10 \\
\mathrm{pm}\end{array}$ & $\begin{array}{l}10 \mathrm{pm}- \\
12 \mathrm{am}\end{array}$ & $\begin{array}{l}12-6 \\
\mathrm{am}\end{array}$ & $\begin{array}{l}6-8 \\
\mathrm{am}\end{array}$ & $\begin{array}{l}8 \mathrm{am}- \\
12 \mathrm{Nn}\end{array}$ & $\begin{array}{l}12 \mathrm{Nn}- \\
2 \mathrm{pm}\end{array}$ & $\begin{array}{l}2-4 \\
\mathrm{pm}\end{array}$ & $\begin{array}{l}\text { Total leaf disc } \\
\text { consumed by 24h }\end{array}$ \\
\hline 50 & 0.5 & 0.5 & 0.25 & 0 & 0 & 0 & 0 & 0 & 0 & 1.25 \\
\hline 100 & 0.25 & 0.25 & 0.25 & 0 & 0 & 0 & 0 & 0 & 0 & 0.75 \\
\hline 150 & 0.25 & 0 & 0 & 0 & 0 & 0 & 0 & 0 & 0 & 0.25 \\
\hline
\end{tabular}

Number of leaf discs consumed by the insect (values are mean $+\mathrm{SE}$ of five).

\section{CONCLUSIONS}

A series of (5-chloro-2-thienyl)(3-substituted phenyl bicyclo[2.2.1]hept-5-en-2yl)methanone derivatives have been synthesized by aqueous phase fly-ash catalyzed DielsAlder [4+2] cycloaddition of cyclopentadiene and aryl $E$ - chalcones. The yields of the methanones are more than $60 \%$.

The antimicrobial, antioxidant and insect antifeedant activities of (5-chloro-2thienyl)(3-substituted phenyl bicyclo[2.2.1]hept-5-en-2-yl)methanones have been evaluated using Bauer-Kirby disc diffusion, DPPH radical scavenging and Dethler's leaf-disc bioassay method. All compounds show satisfactory antimicrobial activities. The halogens, methoxy and nitro substituted compounds shows good antimicrobial activities against their microorganism strains. The compounds containing hydroxy and methoxy substituents shows good antioxidant activity. The compound 3 (3-(3-chlorophenyl) bicyclo[2.2.1]hept-5-en-2yl)(5-chloro-2-thienyl)methanone shows an appreciable antifeedant activity.

\section{ACKNOWLEDGMENT}

The author (Dr. G. T.) Acknowledged the University Grants Commission, New Delhi, India, for financial support Grant no.F.30-23/2011(SA-II), through the UGC-PDF Research Award.

\section{References}

[1] (a) Thirunaryanan G, Q. Science Connect. (2014) DOI: http://dx.doi.org/ 10.5339/ connect.2014.18.

(b) Mubofu E B \& Engberts J B F N, J. Phys. Org. Chem, 17 (2004) 180-186.

[2] Breslow R, Marita, U \& Rideout D C, Tetrahedron Lett, 24 (1983) 1901-1904.

[3] Breslow R \& Marita U, Tetrahedron Lett. 25 (1984) 1239-1240.

[4] Otto S, Bertoncin F \& Engberts J B F N, J. Am. Chem. Soc, 118 (1996)7702-7707.

[5] Otto S \& Engberts J B F N, J. Am. Chem. Soc, 121 (1999) 6798-6806.

[6] Fringuelli F, Piermatti O, Pizzo F \& Vaccaro L, Eur. J .Org. Chem, (2001) 439-455.

[7] Boersma A J, Feringa B L \& Roelfes G, Org. Lett, 9 (2007) 3647-3650. 
[8] Boersma A J, Bruin B, Feringa B L \& Roelfes G, Chem. Commun, 48 (2012) 23942639.

[9] Rideout, D C \& Breslow R, J. Am. Chem. Soc, 102 (1980) 7816-7817.

[10] Otto S., Engberts J B F N, Tetrahedron Lett. 36 (1995) 2645-2648.

[11] Megens R P \& Roefes G, Chem. Eur. J, 17 (2011) 8514-8523.

[12] Jin Q., Zhang L., Cao H, Wang T, Zhu X, Jiang J. \& Liu M., Langmuir 27 (2011) 13847-13853.

[13] Roefes G, Boersma A J \& Feringa B L, Chem. Commun, (2006) 635-637.

[14] Kuo C H, Niemeyer C M \& Fruk L, Croa. Chem. Acta, 84 (2011) 269-275.

[15] Oltra N S \& Roelfes G, Chem. Commun, (2008) 6039-6041.

[16] Otto S, Engberts J B F N \& Kwak J C T, J. Am. Chem. Soc, 120 (1998) 9517-9525.

[17] Boger D L, Lerner R A \& Cravatt B F, J. Org. Chem, 59 (1994) 5078-5079.

[18] Thirunarayanan G, Vanangamudi G, Sathiyendiran V \& Ravi K, Indian J. Chem, 50B, (2011) 593-604.

[19] Thirunarayanan G \& Vanangamudi G, Spectrochim. Acta. 81A, (2011) 390-396.

[20] Thirunarayanan G, Vanangamudi G \& Subramanian M, Org. Chem: An Indian J, 9 (2013) 1-16.

[21] Thirunarayanan G, J. Indian. Chem. Soc, 85, (2008) 447-451.

[22] Thirunarayanan G, Surya S, Srinivasan S, Vanangamudi G \& Sathiyendiran V, Spectrochim. Acta, 75A, (2010) 152.

[23] Vanangamudi G, Subramanian M \& Thirunarayanan G. Arabian. J. Chem, (2013). DOI: 10.1016/j.arabjc.2013.03.006

[24] Dethler's, V. G. Chemical Insect Attractants and Repellents, Blackistan, Philadelphia, (1947) p. 210.

[25] Thirunarayanan G, Mayavel P \& Thirumurthy K, Spectrochim. Acta, 91A (2012) 18-22.

[26] Bauer A W, Kirby W M M, Sherris J C \& Truck M, Am. J. Clin. Pathol, 45 (1966) $493-$ 496.

[27] Vanangamudi G, Subramanian M \& Thirunarayanan G. Arabian. J. Chem, (2013). DOI: 10.1016/j.arabjc.2013.03.006 